УДК $532.13 ; 53.072 .4$

\title{
Орлов Е.В.
}

Одесский национальный экономический университет, E-mail:orlov_ev@onu.edu.ua

\section{Сдвиговая вязкость суспензий твердых сферических частиц}

\begin{abstract}
Проведен анализ зависимости сдвиговой вязкости суспензии сферических частии от их объемной доли. Рассмотрены случаи бесконечно разбавленных, полуразбавленных и концентрированных суспензий. Предложена оценка значений концентрации частиц суспензии, при которой можно не учитывать вклад от гидродинамических взаимодействий. Получена связь между объемной долей частии суспензии и внутренним параметром ячеечных моделей, который характеризует степень межчастичного гидродинамического взаимодействия.
\end{abstract}

Ключевые слова: сдвиговая вязкость, суспензия, объемная доля частиц.

Введение. Задача построения зависимости сдвиговой вязкости суспензии твердых сферических частиц от их объемной доли представляет интерес на протяжении последних ста лет [1]. Важность наличия адекватной экспериментальным данным модели вязкости суспензии твердых сфер заключается в том, что в дальнейшем ее можно будет использовать для описания поведения систем более интересных с прикладной точки зрения. Примером могут служить различные коллоидные системы, электролиты, взвеси заряженных и пористых частиц, полимерные растворы, эмульсии и суспензии частиц несферической формы, а также многие другие системы частиц, имеющих внутреннюю структуру [2].

Существует множество экспериментальных работ по измерению зависимости сдвиговой вязкости суспензии от объемной доли дисперсной фазы $\phi$ (отношение объема, занимаемого всей суспензией к суммарному объему частиц) [3]. Однако точная теоретическая модель, описывающая зависимость вязкости на всем промежутке изменения объемной доли $0<\phi \lesssim 0.64$ пока не получена.

Имеется несколько эмпирических формул, которые согласуются с различными экспериментальными данными при разных значениях объемной доли. В данной работе обсуждается выбор эмпирической формулы, которая лучшим образом соответствует экспериментальным данным. Рассмотрены существующие теоретические модели вязкости суспензии, предложен новый выбор связи объемной доли с внутренними параметрами модели, а также области применимости различных теоретических моделей вязкости.

1. Зависимость вязкости суспензии от объемной доли. Зависимость сдвиговой вязкости суспензии $\eta$ от объемной доли ф была предметом многочисленных экспериментальных исследований и компьютерных симуляций [4]. В результате выяснено следующее: 1) сдвиговая вязкость суспензии твердых сферических частиц зависит от их объемной доли но, в определенных пределах, не зависит от их размера; 2) если рассматривать зависимость вязкости от вели- 


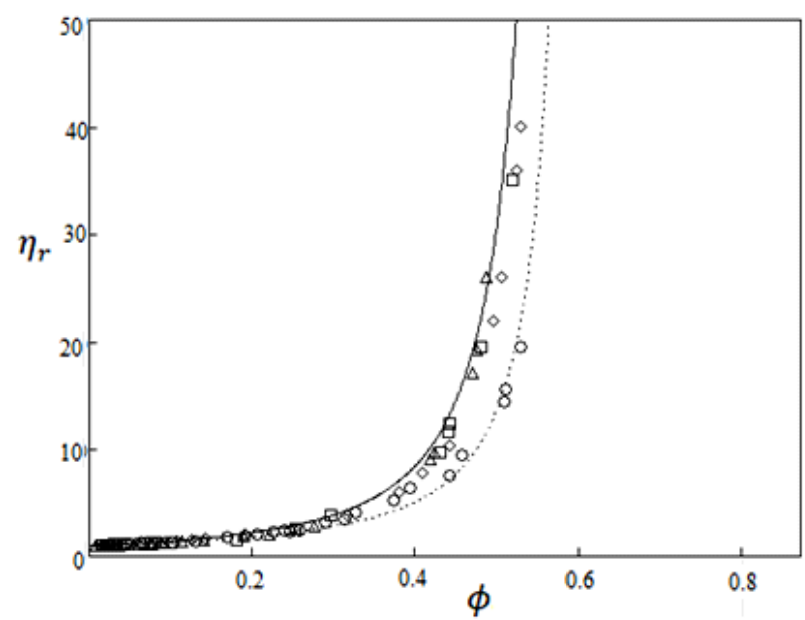

Рис. 1. Зависимость сдвиговой вязкости суспензии от объемной доли. Фигурами представлены измерения для частиц имеющих сферическую форму разных радиусов в пределах от 28 до 110 нм [8]. Сплошная линия соответствует формуле (1), пунктирная формуле (2)

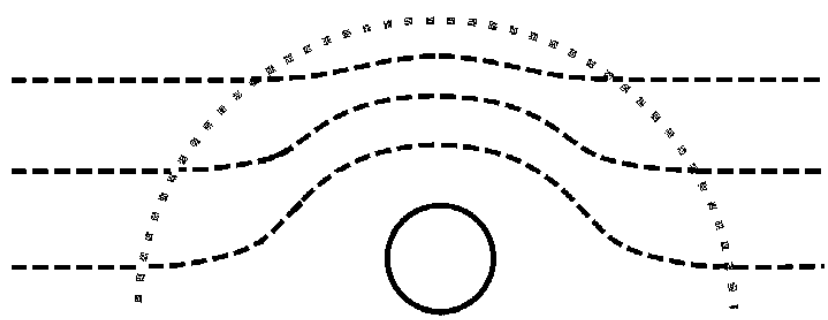

Рис. 2. Область радиуса $r_{*}$ в которой сосредоточено возмущение вызванное присутствием частицы.

чины $\phi / \phi_{\mathrm{m}}$ (где $\phi_{\mathrm{m}}$ - объемная доля, соответствующая максимальной упаковке частиц), то форма кривой будет одинаковой для различных экспериментальных данных; 3) сдвиговая вязкость зависит от степени полидисперсности частиц; 4) если частицы имеют несферическую форму или обладают внутренней структурой, то это в значительной мере сказывается на значении вязкости суспензии. В данной работе рассматриваются неколлоидные суспензии, в которых между частицами существуют только силы гидродинамического взаимодействия.

Для описания зависимости $\eta(\phi)$ было предложено несколько эмпирических выражений. Среди них можно выделить две формулы, результаты которых согласуются с экспериментальными данными практически на все области изменения объемной доли $0<\phi \leq \phi_{m}$. Выражение, предложенное Мароном и Пирсом [5] имеет вид:

$$
\eta_{r}=\frac{\eta}{\eta_{0}}=\frac{1}{\left(1-\frac{\phi}{\phi_{m}}\right)^{2}}
$$

Другая эмпирическая формула предложена Кригером и Догерти [6]

$$
\eta_{r}=\frac{\eta}{\eta_{0}}=\frac{1}{\left(1-\frac{\phi}{\phi_{m}}\right)^{[\eta] \phi_{m}}}
$$

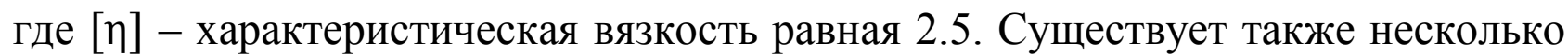
других эмпирических формул [7], однако они либо сводятся к данным двум, либо хуже описывают экспериментальные данные. 
На рис. 1 показана характерная зависимость сдвиговой вязкости от объемной доли, полученная во многих экспериментальных работах и компьютерных симуляциях [8, 9].

В разных работах рассматривались различные значения объемной доли максимальной упаковки сферических частиц. Известно, что случайной упаковке сфер соответствует значение $\phi_{r p}=0.49$, стеклованию $\phi_{g}=0.58$, плотной случайной упаковке $\phi_{r c p}=0.64$, плотной упаковке равных сфер $\phi_{c p}=0.74$. Таким образом, $\phi_{m}$ является подгоночными параметром в данных формулах. Было установлено [10] что, если представить значения измерения вязкости в различных суспензиях от параметра $\phi / \phi_{m}$, то они располагаются практически на одной линии, которая соответствует формуле (1).

Характер зависимости сдвиговой вязкости суспензии твердых сферических частиц в наиболее широком интервале изменения объемной доли описывается выражением (1), однако недостатком этой формулы является ее несогласованность с формулой Эйнштейна

$$
\eta_{r}=\frac{\eta}{\eta_{0}}=1+2.5 \phi
$$

при малых значениях ф. Выражение (2) менее точно описывает экспериментальные данные, однако согласуется с формулой Эйнштейна в пределе бесконечно разбавленных суспензий. Обе эти формулы имеют одинаковую структу-

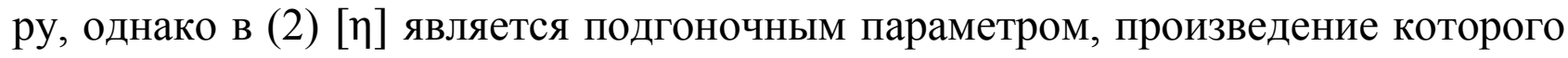
на $\phi_{\mathrm{m}}$ может быть равно 2 , что соответствует формуле (1). Если положить, что $\phi_{m}=0.64$, то $[\eta]=3.1$ что отличается от классического 2.5 . Для $\phi_{m}=0.74$ значение $[\eta]=2.7$, что очень близко к эйнштейновской константе. Таким образом, в дальнейшем будем пользоваться выражением (1) в качестве эмпирической формулы наилучшим образом описывающей экспериментальные данные.

Рассмотрим далее имеющиеся теоретические модели вязкости на различных интервалах изменения объемной доли твердой фазы.

2. Бесконечно разбавленные суспензии. В случае бесконечно разбавленных суспензий предполагается, что взвешенные частицы не взаимодействуют между собой. Впервые этот случай был рассмотрен Эйнштейном [11], который получил выражение (3) для вязкости суспензии твердых сферических частиц. Им были использованы уравнения Стокса для движения сферы и при помощи соображений о диссипации энергии получено выражение, которое в дальнейшем было принято классическим предельным случаем, когда значения объемной доли стремятся к нулю. В дальнейшем, несмотря на критику [12], это выражение считалось точным в подавляющем количестве работ по моделированию сдвиговой вязкости суспензий сферических частиц, а иногда и намного более сложных систем.

В зависимости от оборудования и техники проведения эксперимента точность измерений сдвиговой вязкости суспензии достигает 2-3\% [3]. Определим, на каком расстоянии $r_{*}$ от центра частицы квадрат возмущения поля ско- 
рости будет отличаться от исходного на 3\% (рис. 1). Решение задачи Стокса в сферической системе координат для движущейся сферы имеет вид

$$
\begin{gathered}
v_{r}=\frac{1}{2} u \cos \theta\left(\frac{3 a}{r}-\frac{a^{3}}{r^{3}}\right), \\
v_{\theta}=-\frac{1}{4} u \sin \theta\left(\frac{3 a}{r}+\frac{a^{3}}{r^{3}}\right),
\end{gathered}
$$

где $a$ - радиус частицы, $u$ - скорость движения частицы. Пренебрегая членами высших порядков, получим

$$
\frac{|v|^{2}}{u^{2}} \approx \frac{9}{16}\left(\frac{a}{r}\right)^{2}\left(3 \cos ^{2} \theta-1\right)
$$

В направлении движения частицы $(\theta \approx 0)$ должно выполняться следующее равенство $\frac{9}{8}\left(\frac{a}{r_{*}}\right)^{2}=0.03$, откуда $r_{*} \cong 6.1 a$. Таким образом, при построении вязкости суспензии эффектами гидродинамического взаимодействия можно пренебречь в том случае, когда среднее межчастичное расстояние в шесть раз превышает радиус частицы. Такому среднему межчастичному расстоянию соответствуют значения объемной доли $\phi \approx 0.01$. Обычно полагают, что формула Эйнштейна применима для значений $\phi \approx 0.1$. Однако вышеприведенная оценка показывает, что при подобных значениях объемной доли уже необходимо учитывать гидродинамическое взаимодействие частиц, т.е. строго говоря, использования формулы (3) не обосновано.

С другой стороны при $\phi \approx 0.01$ погрешность измерения вязкости не позволяет получить точного значения характеристической вязкости [ๆ], которая является тангенсом угла наклона линейной зависимости в нуле. На рис. 3 приведены три прямые, угловые коэффициенты которых соответственно равны 2, 2.5 и 3.1. Видно, что сделать заключение о равенстве характеристической вязкости эйнштейновскому значению 2.5 было бы не верно. Более того, сама модель суспензии твердых сферических частиц не претендует на столь точное описание суспензии реальных частиц.

3. Полуразбавленные суспензии. Для полуразбавленных суспензий значения объемной доли находятся в интервале $\phi \lesssim 0.25$ [13]. В этой области значений фнеобходимо учитывать гидродинамические взаимодействия. Взаимодействия между двумя частицами были рассмотрены Бетчелором [14], между тремя Чихоцким [15]. Подобные вычисления приводят к выражению вида

$$
\eta_{r}=1+B_{1} \phi+B_{2} \phi^{2}+B_{3} \phi^{3}+\ldots
$$

где обычно полагают $B_{1}=2.5$. Для коэффициента $B_{2}$ в различных работах были получены значения в пределах $5.2 \leq B_{2} \leq 14.1$. Значение $B_{3}=9.1$ [15]. Из рис. 4 видно, что наиболее точно полученное значение $B_{2}=6.2$ дает возможность получить согласие с экспериментом до значений $\phi \lesssim 0.15$. Однако, если использовать полученное в работе [12] значение $B_{2}=14.1$, то удается добиться 


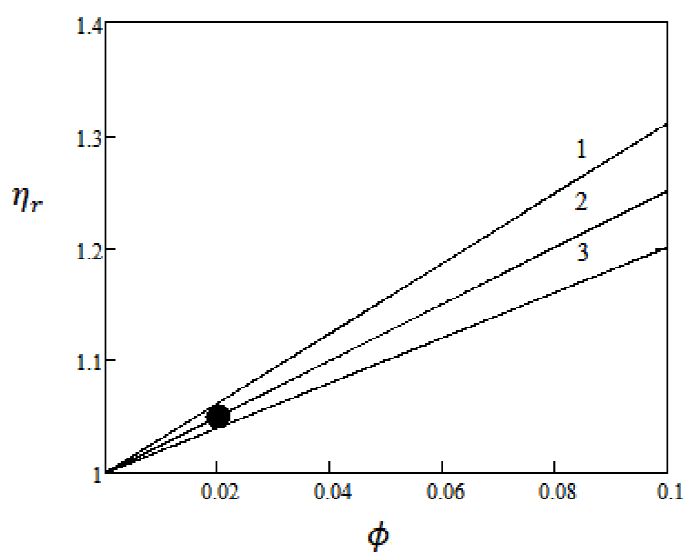

Рис. 3. При столь малых значениях $\phi \approx 0.01$, получить точное значении угла наклона прямой не представляется возможным: $1-[\eta]=3.1 ; 2-[\eta]=2.5$; $3-[\eta]=2$.

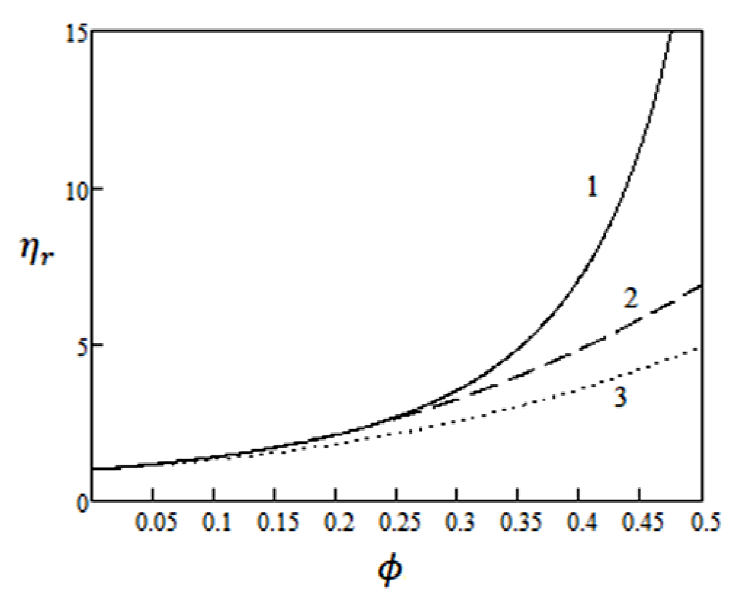

Рис 4. Зависимость вязкости от объемной доли: 1 - экспериментальная зависимость (1); 2 - выражение (4) с $B_{2}=14.1 ; 3$ - выражение (4) с $B_{2}=5.2$.

соответствия до значений $\phi \lesssim 0.25$. Таким образом, существующая теория двух и трех частичных гидродинамических взаимодействий позволяет адекватно описать экспериментальную зависимость вязкости суспензии от объемной доли частиц.

4. Концентрированные суспензии. Полиномиальные формулы вида (4) не могут адекватно описать реальную зависимость вязкости от объемной доли, так как они предсказывают конечные значения вязкости при $\phi \rightarrow \phi_{m}$. Наибольшее возможное значение объемной доли соответствует плотной упаковке равных сфер, при котором $\phi=0.74$ (значение вязкости равно бесконечности). Однако максимальное значение объемной доли частиц суспензии намного меньше. Если суспензия моделируется как жидкая среда с растворенными в ней жесткими частицами, то $\phi_{m}$ должно соответствовать случайной упаковке твердых сфер $\phi_{m}=0.49$. При больших значениях объемной доли суспензия больше похожа на простую жидкость со смазкой между частицами и гидродинамические представления в данном случае не применимы.

Для моделирования вязкости в области изменения $0.25 \lesssim \phi \lesssim 0.49$ используют ячеечные модели. Наиболее известны две из них, это модели Симхи [16] и Хаппеля [17]. Полагается, что вся суспензия разбита на ячейки, в центре каждой из которых находится сферическая частица. Гидродинамическая задача ставится для одной ячейки, при этом влияние остальных ячеек обусловлено выбором граничных условий на внешней поверхности ячейки. В модели Симхи возмущение поля скоростей на границе ячейки равно нулю. В модели Хаппеля возмущение тензора напряжений на границе ячейки равно нулю.

Во всех ячеечных моделях возникает важный параметр $\psi=\left(\frac{a}{R}\right)^{3}$, где $R-$ радиус ячейки, который связан с экспериментально измеряемой объемной до- 
лей, $a$ - радиус частицы. Существует несколько вариантов выбора зависимости $\psi=\psi(\phi)$. В большинстве работа полагается

$$
\psi=\phi
$$

Однако так как ячейки имеют сферическую форму то, в этом случае, не учитывается область, занимаемая жидкостью между ячейками. Получим более обоснованную с геометрической точки зрения зависимость радиуса ячейки от объемной доли.

Во-первых, так как жидкость является неупорядоченной системой, то максимальное значение объемной доли должно быть равно значению при случайной упаковке равных сфер. Во-вторых, при любой концентрации частиц суспензии в определенном ее объеме находится столько же ячеек, сколько и частиц, при этом сферические ячейки всегда занимают долю всего объема, соответствующую случайной упаковке, т.е. $\phi_{r c p}=0.49$. Таким образом, получим следующее выражение

$$
\frac{V_{s c}}{V}=\phi_{r c p}
$$

где $V$ - объем всей суспензии, $V_{s c}$ - объем, занимаемый всеми ячейками. Учитывая, что

$$
\phi=\frac{V_{s p}}{V}
$$

где $V_{s p}$ - объем, занимаемый всеми частицами, получим:

$$
\phi=\frac{V_{s p}}{V_{s c}} \phi_{r c p}=\frac{n V_{p}}{n V_{c}} \phi_{r c p}=\frac{a^{3}}{R^{3}} \phi_{r c p}=\psi \phi_{r c p},
$$

$n$ - концентрация частиц. Полученная новая зависимость

$$
\psi=\frac{\phi}{\phi_{r c p}}
$$

обладает тем свойством, что $\phi_{r c p}$ появляется в ячеечной модели не как параметр, а как следствие геометрии рассматриваемой системы. В других моделях и эмпирических формулах вида (2) эта величина являлась подгоночным параметpoм.

На рис. 5 приведены результаты ячеечных моделей Симхи и Хаппеля в предположении различных выборов внутреннего параметра $\psi$. При выборе зависимости (5) результаты обеих моделей количественно не согласуются с экспериментальными данными. При этом наиболее часто используемая модель Симхи приводит к завышенным результатам и даже качественно не согласуется с экспериментальными данными. Если же выбрать более адекватную с геометрической точки зрения зависимость (6), то из рис. 5 видно, что данные модели не в состоянии описать поведение сдвиговой вязкости концентрированных суспензий даже на качественном уровне. На наш взгляд это является следствием того, что выбранный тип поступательного движения частицы в обеих моделях не может адекватно описать гидродинамические потоки, присутствующие в реальных суспензиях.

При $0.49 \lesssim \phi<0.64$, когда превышен предел случайной упаковки твердых сфер, суспензию нельзя описывать при помощи гидродинамических моде- 


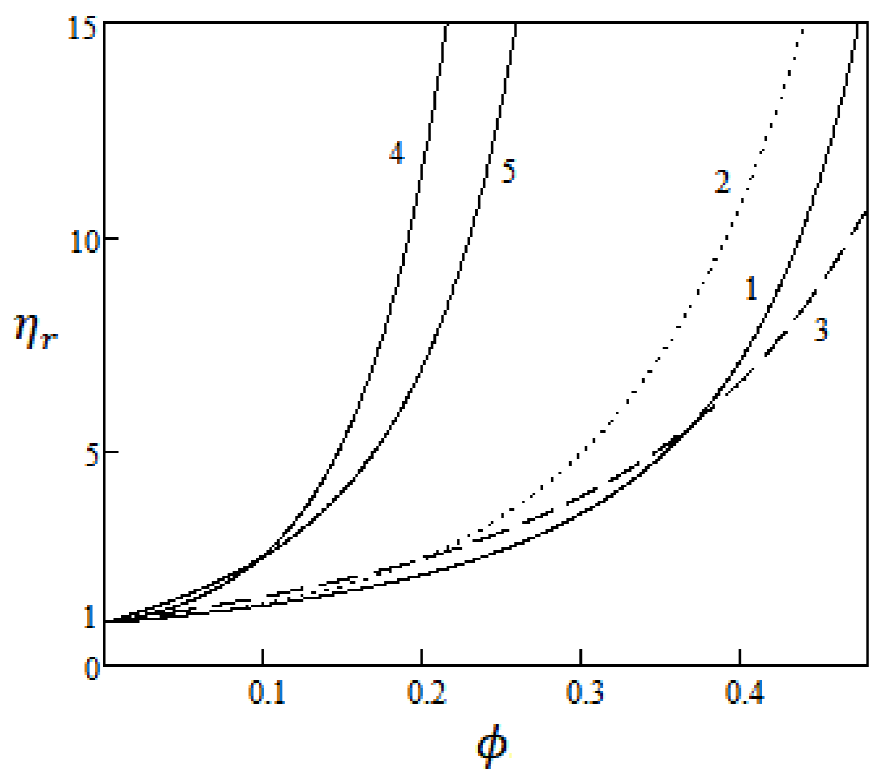

Рис. 5. Зависимость вязкости от объемной доли: 1 соответсвует формула (1); 2 - формула Симхи при зависимости (5); 3 - формула Хаппеля при зависимости (5); 4 - формула Симхи при зависимости (6); 5 - формула Хаппеля при зависимости (6).

лей, т.к. гидродинамические потоки жидкости между частицами и граничные условия не оказывают существенного влияния на вязкость суспензии. Это подтверждается тем, что форма экспериментально полученной зависимости вязкости от объемной доли в данном случае подобна зависимости для простых жидкостей. Межчастичная жидкость играет роль смазки, что отличает концентрированные суспензии от гранулированных сред. Следовательно, требуются новые теоретические модели для описания подобных систем.

Таким образом, существующие модели сдвиговой вязкости концентрированной суспензии не в состоянии адекватно описать экспериментальные данные на всей области изменения объемной доли. Если же выбрать параметр $\psi$ ячеечных моделей согласно ее геометрии, то экспериментально полученная зависимость $\eta(\phi)$ не воспроизводится даже на качественном уровне.

Выводы. Существующие экспериментальные работы по моделированию поведения суспензии твердых сферических частиц показывают не полную согласованность полученных данных. Это объясняется тем, что исключить посторонние факторы, оказывающие влияние на вязкость суспензии достаточно сложно. Однако вид зависимости сдвиговой вязкости суспензии от объемной доли частиц наиболее приближен к выражению (1).

Показано, что область применимости модели Эйнштейна сдвиговой вязкости бесконечно разбавленной суспензии намного более узкая, чем считалось прежде. Таким образом, проверка адекватности формулы Эйнштейна приводит к значительным техническим сложностям.

Для описания поведения сдвиговой вязкости концентрированных коллоидных систем широко используют ячеечные модели. При этом за основу выби- 
рают одну из двух ячеечных моделей, описывающих суспензию твердых сферических частиц. Показано, что 1) сами эти модели не вполне адекватно описывают экспериментальные данные; 2) выбор внутреннего параметра в обоих моделях не согласуется с геометрией системы; 3) если выбрать внутренний параметр системы адекватно геометрии, то результаты не согласуются с экспериментальными данными даже на качественном уровне.

\title{
Литература:
}

1. Mewis J., Wagner N.J. Colloidal suspension rheology. - Cambridge university press, 2012. - 393 p.

2. Babick F. Suspensions of colloidal particles and aggregates. - Springer, 2016. -340 p.

3. Хорольский О.В., Руденко О.П. // Український фізичний журнал. - 2015. - Т. 60. № 9. - C. 882.

4. Laganapan A.M.K. et al. // Journal of Chemical Physics. - 2015. - Vol. 142. №14. - P. 144101.

5. Maron S.H., Pierce P.E. // Journal of Colloid Science. - 1956. - Vol. 11. №1. - P. 80.

6. Krieger I.M. // Advances in Colloid and Interface Science. - 1972. - Vol. 3. №2. - P. 111.

7. Thomas D.G. // Journal of Colloid Science. - 1965. - Vol. 20. №3. - P. 267.

8. van der Werff J.C., de Kruif C.G. // Journal of Rheology. - 1989. - Vol. 33. №3. - P. 421.

9. Sivadasan V. et al. // Physics of Fluids. - 2019. - Vol. 31. №10. - P. 103103.

10. Allain C., Cloiter M., Lacoste B., Marsone I. // Journal of Chemical Physics. - 1994. Vol.100. №6. - P. 4537.

11. Einstein A. // Annals of Physics. - 1906. - Vol. 19. - P. 289.

12. Хаппель Дж., Бреннер Г. Гидродинамика при малых числах Рейнольдса. / Пер. с англ. Бермана В.С., Марокова В.Г. - М.: Мир, 1976. - 630 с.

13. Mueller S., Llewellin E.W., Mader H.M. // Proceedings of the Royal Society A - 2010. Vol. 466. №2116. - P. 1201.

14. Batchelor G.K., Green J.T. // Journal of Fluid Mechanics - 1972. - Vol. 56. № 3. - P. 401.

15. Cichocki B., Ekiel-Jezewska M.L., Wajnryb E. // Journal of Chemical Physics. - 2003. Vol.119. №1. - P. 606.

16. Simha R. // Journal of Applied Physics. - 1952. - Vol. 23. №9. - P. 1020.

17. Happel J. // Journal of Applied Physics. - 1957. - Vol. 28. №11. - P. 1288.

\section{Orlov E.V. \\ Shear viscosity of hard sphere suspensions}

\begin{abstract}
SUMMARY
The dependence of the shear viscosity of spherical particles suspension on the volume fraction is analyzed. Existing experimental works on modeling the behavior of a suspension of solid spherical particles show incomplete agreement between the data obtained. This is because it is quite difficult to exclude various factors that affect the viscosity of the suspension. It is rather difficult to obtain particles which shape is closest to spherical. Eliminating all types of interactions inherent in colloidal systems is also a difficult task. However, it can be
\end{abstract}


assumed that the form of the dependence of the shear viscosity of the suspension on the volume fraction of particles has a certain shape typical of a suspension of hard spheres.

The entire range of volume fraction changes from 0 to 0.64 is considered. An estimate of the region of variation in the volume fraction of the dispersed phase is proposed, at which hydrodynamic interactions can be ignored. It is shown that the range of applicability of the Einstein model of shear viscosity of an infinitely diluted suspension is much narrower than previously thought. Thus, checking the adequacy of the Einstein formula leads to significant technical difficulties.

Cell models are widely used to describe the shear viscosity behavior of concentrated colloidal systems. In this case, one of two cell models describing a suspension of solid spherical particles is selected as the basis. A relationship between the volume fraction of suspension particles and the internal parameter of cell models, which characterizes the degree of interparticle hydrodynamic interaction, is obtained. It was shown that 1) these models themselves do not adequately describe the experimental data; 2) the choice of the internal parameter in both models is not consistent with the geometry of the system; 3) if we choose the internal parameter of the system adequately to the geometry, then the results do not agree with the experimental data even at a qualitative level.

Key words: shear viscosity, suspension, volume fraction of particles.

\section{Орлов С.В. \\ Зсувна в'язкість суспензї твердих сферичних частинок}

\section{АНОТАЦІЯ}

Проведений аналіз залежності зсувної в'язкості суспензї сферичних частинок від їх об'ємної долі. Існуючі експериментальні роботи по моделюванню поведінки суспензіі твердих сферичних частинок показують не повну узгодженість отриманих даних між собою. Це пояснюється тим, щุо виключити різні чинники, щุо роблять вплив на в'язкість суспензї досить складно. Отримати частинки, форма яких найбільш наближена до сферичної досить важко. Виключити всі види взаємодій властиві колоїдним системам також є складним завданням. Проте можна вважати, що вигляд залежності зсувної в'язкості суспензї від об'ємної долі частинок має певну форму, характерну для суспензії твердих сферичних частинок між якими існують лише гідродинамічні взаємоdii.

Розглянутий весь інтервал зміни об'ємної долі від 0 до 0.64. Запропонована оиінка області зміни об'ємної долі дисперсної фази, при якій можна не враховувати гідродинамічні взаємодії. Показано, що область застосування моделі Айнштайна зсувної в'язкості нескінченно розбавленої суспензї̈ набагато вужча, ніж вважалося раніше. Таким чином, перевірка адекватності формули Айнштайна приводить до значних технічних складношів.

Для опису поведінки зсувної в'язкості конщентрованих колоїдних систем широко використовують коміркові моделі. При цьому за основу вибирають одну з двох коміркових моделей, щзо описують суспензію твердих сферичних частинок. В роботі отриманий зв'язок між об'ємною долею частинок суспензії $і$ внутрішнім параметром коміркових моделей, який характеризує міру міжчасткової гідродинамічної взаємодії. Показано, щуо 1) самі иүі моделі не цілком адекватно описують експериментальні дані; 2) вибір внутрішнього параметра в обох моделях не узгоджується з геометрією системи; 3) якщзо вибрати внутрішній параметр системи адекватно геометрії, то результати не узгоджуються з експериментальними даними навіть на якісному рівні.

Ключові слова: зсувна в'язкість, суспензія, об'ємна частка частинок. 